\title{
Polar curved polycyclic aromatic hydrocarbons in soot formation
}

\author{
Jacob W. Martin ${ }^{\mathrm{a}}$, Kimberly Bowal ${ }^{\mathrm{a}}$, Angiras Menon ${ }^{\mathrm{a}}$, Radomir Slavchov ${ }^{\mathrm{a}}$, Jethro Akroyd ${ }^{\mathrm{a}}$, Sebastian Mosbach ${ }^{\mathrm{a}}$, \\ Markus Kraft ${ }^{\mathrm{a}, \mathrm{b}, *}$ \\ ${ }^{a}$ Department of Chemical Engineering and Biotechnology, University of Cambridge, West Site, Philippa Fawcett Drive, Cambridge, CB3 OAS, \\ United Kingdom \\ ${ }^{b}$ School of Chemical and Biomedical Engineering, Nanyang Technological University, Singapore 637459
}

\begin{abstract}
Recent experimental and computational work suggests that a significant number of curved aromatic molecules are found in carbon materials such as soot. These curved aromatic molecules contain a substantial dipole moment (4-6.5 debye for the sizes seen in soot molecules containing 10-20 rings). In this paper we employ electronic structure calculations to determine the earliest onset of curvature integration. We found an interplay between the $\sigma$ bonding inducing curvature and the $\pi$ bonding resisting curvature with a size of six rings, with at least one being pentagonal, required to curve an aromatic molecule. The interactions between polar curved arenes and other species are then considered with particular focus on nascent soot formation, indicating that heterogeneous, not homogeneous, nucleation could be a significant factor in soot formation.
\end{abstract}

Keywords:

Curved polycyclic aromatic hydrocarbons, Flexoelectric dipole, Soot formation, Combustion

*Supplementary Material is available for this paper.

\section{Introduction}

Carbon particulate emissions from incomplete combustion of hydrocarbons in engines and open fires contributes to climate change[1,2] and impacts human health[3]. Considerable efforts to understand and mitigate the formation of soot has led to understanding of the gas phase chemistry and the dynamics of soot particles as they agglomerate. However, significant uncertainty remains around the transition from molecules into a solid particle[4]. Recent high resolution transmission electron microscopy (HRTEM) studies have shown that significant fractions of aromatic molecules in soot contain non-hexagonal rings[5, 6]. Curvature analysis of the fringes using geometrical [5] and statistical models of the curvature [7] showed that the majority of fringes contained a single inflection point indicating pentagon integration, with $28-49 \%$ of the fringes containing curvature indicating $>1$ pentagon. The range of pentagon integration was predicted to span 1-3 pentagons. Corannulene[8] and fullerenes[9] have also

\footnotetext{
${ }^{*}$ Corresponding author

Email address: mk306@cam.ac.uk (Markus Kraft)
}

been synthesised in flames, so the presence of these curved fragments does not come as a surprise.

Once curvature is integrated, significant electric polarisation of the molecule occurs[10]. For the size of fragments found in primary soot particles determined from HRTEM and optical band gap methods (10-20 rings)[11] we predicted a range of 4-6.5 debye (which is two to three times that of water; 1.85 debye[12])[10]. Figure 1 shows the electric potential around water,

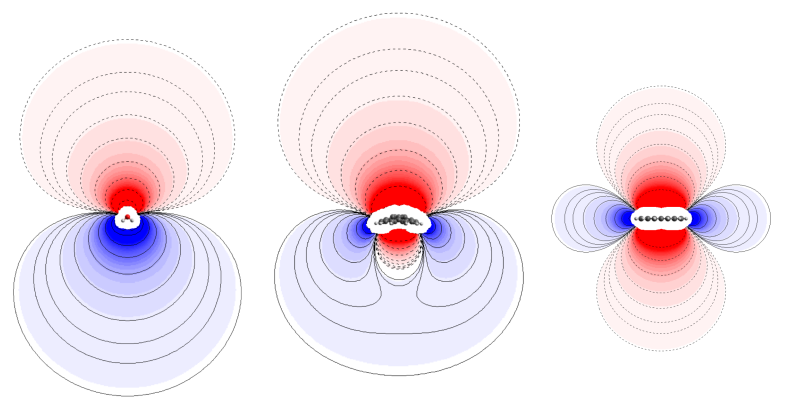

Figure 1: Cross section of the electric potential, $(+)$ blue, $(-)$ red, for water (left), corannulene (middle) and coronene (right). 
corannulene and coronene. The quadrupolar potential of coronene with the negative potential, perpendicular to the molecule's plane, can be clearly seen and is due to the $\pi$-bonding. This becomes significantly polarised for corannulene, giving a dipole moment similar in magnitude to water.

In this paper we will outline some of the potential impacts of these curved fragments in soot. We begin by discussing the earliest onset of curvature integration, then calculate the binding energy between dimers of curved PAH. The interaction between polar molecules, non-polar molecules and chemi-ions are then discussed as they relate to nascent soot formation.

\section{Methodology}

The electronic structure calculations and geometry optimisations were performed using the Gaussian 09 software[13]. For the curvature integration calculations, the hybrid density functional theory (DFT) method B3LYP with the 6-311G(d,p) basis set was used, which has been found to provide structures for small curved aromatics to the quality of those determined from x-ray crystallography[14, 15]. We found this level of theory to provide molecular dipoles moments to within $6 \%$ error compared with experimentally measured dipole moments of corannulene[10]. To guarantee curvature integration could be confirmed, care was taken to ensure that geometry optimisations reached the global minimum. $\mathrm{C}_{1}$ symmetry was enforced for all structures, initial geometries were purposely curved away from planar and frequency calculations ensured no negative frequencies indicating saddle points in the potential energy. For flat/planar PAH dimerisation calculations we have previously made use of symmetry-adapted perturbation theory DFT methods SAPT(DFT) which were found to reproduce the exfoliation energies of graphene[16]. In this case the intermolecular potential energy was easily sampled by increasing the interplanar distance between the dimers and calculating the binding energy as a function of distance. However, for curved aromatic molecules a more complex geometry can result from the symmetry breaking of the curvature and full geometryoptimised structures were sought. This required a sufficiently rapid and scaleable method in order to optimise the geometry of the largest structures. We therefore made use of a hybrid DFT method with an empirical dispersion correction B97D[17]. This method has been shown to produce binding energy to accuracies of CCSD(T) or SAPT(DFT) methods for corannulene and sumanene[18, 19]. (See Supplementary Information for further details.)

\section{Results and discussion}

\subsection{Curvature integration}

We have previously found a direct relationship between the curvature of $\mathrm{sp}^{2}$ hybridised carbon atoms (measured via pyramidalisation angle $\theta_{\mathrm{p}}$ ) and the local dipole moment in curved PAH (through the flexoelectric constant $\left.f_{\theta \mathrm{p}}=2.24 \mathrm{D} / \mathrm{rad}\right)$. This pyramidalisation angle $\theta_{\mathrm{p}}$ describes the amount of local curvature at each carbon atom and is constructed from a vector that makes equal angles with the three sigma bonds $\theta_{\sigma \pi}$ $\left(\theta_{\mathrm{p}}=\theta_{\sigma \pi}-90^{\circ}\right)$, which is used to describe when a structure becomes curved (when $\theta_{\mathrm{p}}>0$ ).

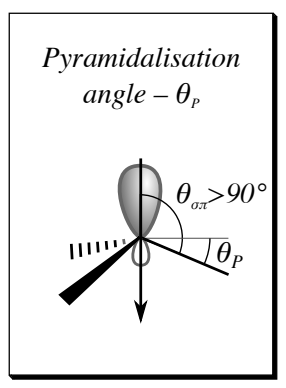

Figure 2: Schematic of the pyramidalisation angle and the definition of $\theta_{p}$ and the equal angles of $\theta_{\sigma \pi}$

It is important to understand the onset of curvature integration in PAH formation in order to know when a significant flexoelectric dipole moment is expected during PAH formation in hydrocarbon flames. Various mechanisms for pentagon integration have been proposed, such as acetylene addition to fluoranthene,[20] dehydrogenation of a five-member bay site,[21-23] oxidation of a zig-zag edge,[24] dehydrogenation of aromers[25] or a $C_{1} / C_{2}$ mechanism[26]. Our goal here is not to comment on which mechanisms were of most importance, but to consider under which molecular geometries curvature is integrated and expand the structures explored in [10]. Figure 3 shows the various strategies employed.

The geometry of hexagonal and pentagon nets should dictate that once two hexagonal rings border a pentagonal ring, curvature should be integrated. Considering the base structures in Figure 3 ac, fa, ph, be and 1a no curvature is observed until the pentagon is entirely enclosed by five hexagonal rings. To understand this discrepancy between the geometrical model and that found in the calculated structures, the bonding must be considered. The $\sigma$-bonding of the $\mathrm{sp}^{2}$ aromatic network contributes to curvature integration by limiting the bond lengths (1.3-1.6 $\AA$ ) and providing an angle potential optimal at $120^{\circ}$. The $\pi$-bonding network how- 


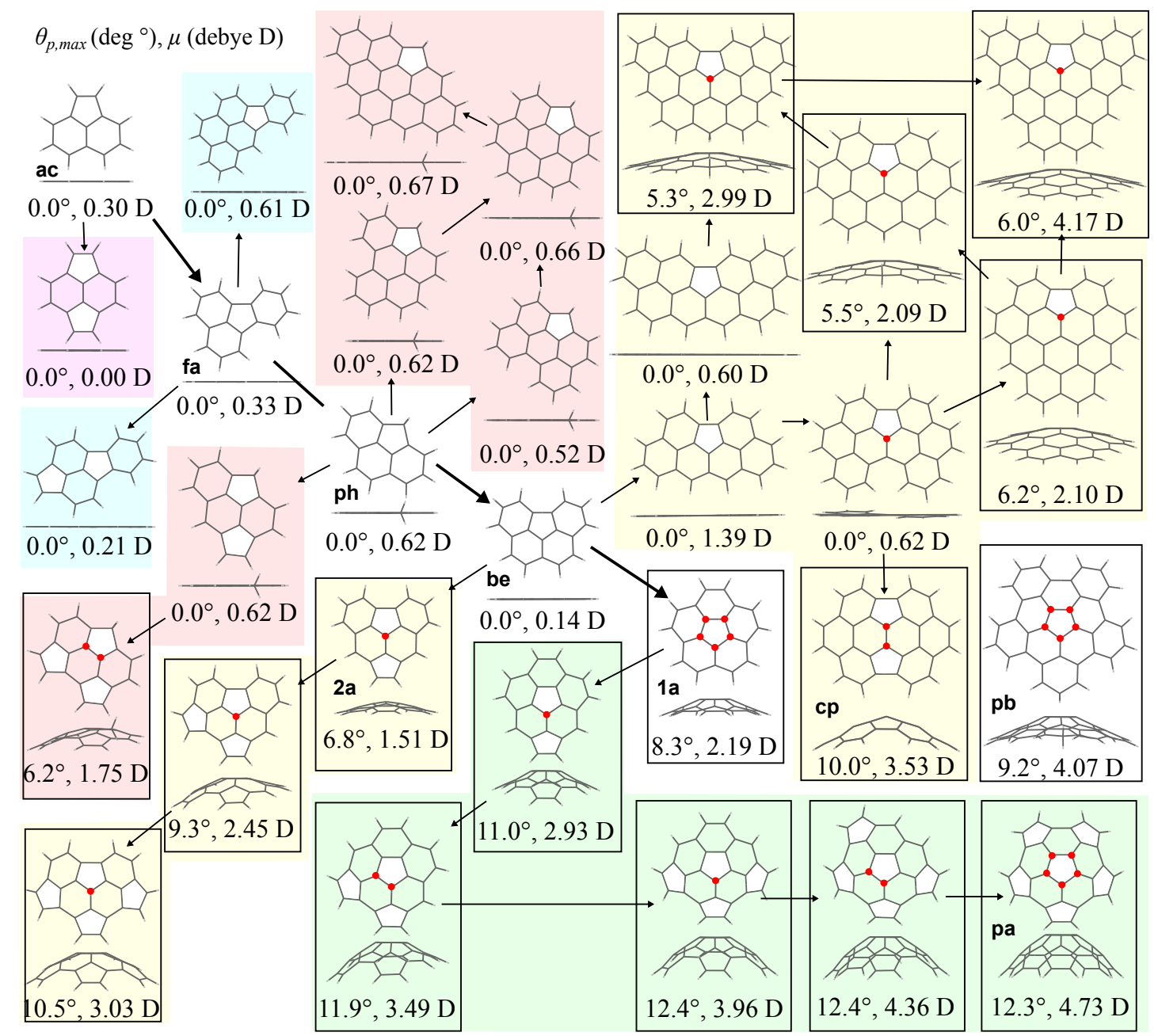

Figure 3: Initial structures with single pentagons with two ac, three fa, ph, four be or five 1a bordering hexagons are shown such as hexagonal extension ii) top right side, and acetylene addition iii) bottom left. Optimised geometries are shown from above and side. The curved structures are framed by a black box. The most pyramidalised carbon atom of each structure is denoted by a red circle and its' value is quoted underneath, along with the dipole moment calculated at the B3LYP/6-311G(d,p) level of theory.

ever resists bending (leading to a large elastic modulus $\left.21.5 \mathrm{eV} / \AA^{2}[27]\right)$, which favours the planarisation of the molecules. The $\pi$-bonding dominates for two to four bordering hexagons with the bond lengths and angles changing to accommodate the planar structure. The planarisation is only overcome for the structure with a pentagon completely enclosed by hexagons $\mathbf{1 a}$.

We extended our previous set of structures that integrated curvature due to enclosed pentagons i) to include non-enclosed pentagons to determine other curved geometries via two methods: increasing the hexagonal aromatic network around the structure ii) or by acetylene addition to zig-zag edge site iii), as suggested by Pope and Howard[20]. For structures fa and ph with a single pentagon and three bordering hexagons, additional hexagonal network growth did not give rise to curvature. However, for the structure be with four hexagonal rings enclosing the pentagonal ring, we found that hexagonal growth did lead to curvature and a flexoelectric dipole moment. However, substantial growth was required with initial addition of hexagonal aromatic rings to the left and right of the pentagonal ring not leading to curvature integration; hexagonal rings were required a further layer below the be molecule (as observed in the orientation in Figure 3). We also found that substantial curvature could be integrated for the geometry with two unenclosed pentagons joined by two hexagons (Figure $3 \mathbf{c p}$ ). An effective curvature in- 


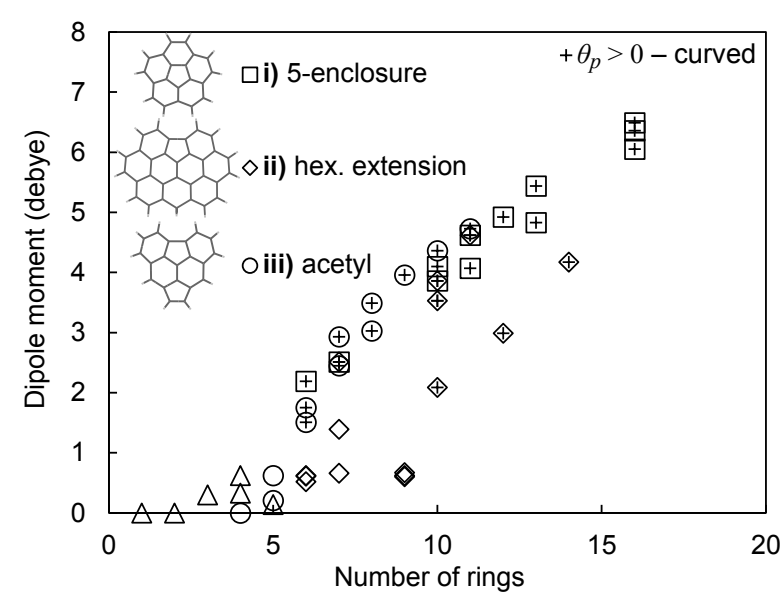

Figure 4: Dipole moment as a function of the number of rings for different curvature integration strategies with curved structures indicated by a plus symbol.

tegration mechanism was also found for the addition of acetylene to benzo(ghi)fluoranthene be. This rapidly increased the curvature (from $\theta_{\mathrm{p}, \max }=6.8,9.3,10.5^{\circ}$ ) and dipole moment of these structures (from $\mu=1.51,2.45$ to 3.03 debye). This was also explored for corannulene and a substantial dipole moment found with these nonintegrated pentagonal rings giving dipole moments of $2.93,3.49,3.96,4.36$ to 4.73 debye. The additional pentagonal ring integrated curvature more effectively due to its decreased internal angles compared with the hexagonal rings. This is further highlighted by comparing the curvature of pentacetylcorannulene pa and pentabenzocorannulene $\mathbf{p b}$, the former was found to be more pyramidalised $-\theta_{\mathrm{p}, \max }=12.3^{\circ}$ compared with the later $\theta_{\mathrm{p}, \max }=9.2^{\circ}$. Acetylene additions to zig-zag sites - acetyl groups are unstable at high temperature and are prone to migrate on hexagonal edges or desorb[21]. This indicates that the complete enclosure of a pentagon by hexagonal rings i) would represent the most effective manner to integrate stable curvature, with the second most effective being acetylene addition to zig-zag edge site iii).

Returning to the question of the critical size for the onset of curvature Figure 4 shows the dipole moment as a function of ring size with the onset of curvature found to be 6 rings for the pentagon enclosure and acetyl curvature integration strategies. For the hexagonal extension strategy the onset of curvature was found to be at 10 rings.

\subsection{Interactions between $\mathrm{CPAH}$}

We will now consider the impact of the flexoelectric dipole moment on the dimerisation of curved aromatic dimers in the size range found in soot (4-20 rings) with the most stable pericondensed species having pentagonal rings entirely enclosed by hexagons i). The dimerisation energy has been used to consider the stability of clusters of flat PAH molecules previously.[4, 2830] Figure 5 shows the various binding energies of curved aromatic homodimers compared with flat PAHs of pyrene, coronene, ovalene, hexabenzocoronene and circumcoronene taken from [29]. For structures containing one to two pentagonal rings, we found a similar binding energy compared with planar PAH. However, further increases in the number of pentagons decreased the binding energy compared with a flat PAH of a similar mass.

Comparing corannulene and coronene with similar binding energies, the role of the dispersion interactions and dipole can be deduced. SAPT(DFT) calculations show a reduced dispersion interaction between corannulene dimers of $-136 \mathrm{~kJ} / \mathrm{mol}$ [19] compared with -145 $\mathrm{kJ} / \mathrm{mol}[31]$ for coronene. This is also seen in the greater intermolecular distance between the dimers of corannulene of $3.62 \AA$ compared with $3.58 \AA$. The role of the dipole can be seen in the electrostatic contribution, which for corannulene is $22 \%$ of the attractive potential compared with $13 \%$ for coronene. We also observed a higher dipole moment for the dimers compared with the sum of the two static dipole moments, indicating a small contribution from induction. SAPT(DFT) found induction to make up $4 \%$ of the attractive force in the case of corannulene[19]. (See the Supplementary Information for full details with comparisons to B97D simulations.)

While the dipole moment contributes to the binding energy, the curvature decreases the dispersion interactions due to steric effects. This can be seen from an increased intermolecular distance (see Supplementary Information) from 4-4-5 $\AA$ for one to three pentagons increasing to $\sim 6-7 \AA$ for four or more pentagons integrated. Due to the short range of the dispersion interactions (scaling as $\propto 1 / r^{6}$ ) this increased distance significantly decreases the binding energy, most evidently for the structures with three or more pentagonal rings. For the highly strained structures a tilt was also found for the geometries. This is due to the positive hydrogen atoms interacting with the negative charge concentration around the carbon at the rim of the bowl and has been observed in crystal structure.[32]

The similar binding energy of cPAH with one to two pentagonal rings to planar PAH indicates that homogeneous nucleation is unlikely to occur at flame temperatures[29]. Some preliminary molecular dynamics simulations have been performed on dimers $3 \mathbf{c}$ by Violi and a similar clustering behaviour was seen com- 
pared with planar PAH. This is consistent with corannulene's sublimation temperature at standard conditions of $640 \mathrm{~K}$ [33]), which is similar to perylene $660 \mathrm{~K}$ at [34], a planar PAH with the same number of carbon atoms.

We have previously found that flat PAHs form stacked structures with strong dispersion interactions that are not significantly porous[35]. For species with greater than three pentagonal rings, significant decreases in the dispersion interactions could increase the porosity of soot. Soot with significant curvature, measured using thermal gravimetry, was found to oxidise at lower temperatures than more graphitic soot, possibly indicating a more accessible structure[5]. However, it is unclear whether this was primarily due to the increased porosity or an increased reactivity of curved structures due to the decrease in the aromaticity from the integration of non-hexagonal rings[36]. This decreased aromaticity provides a greater electron density to the carbon atoms around the rim of curved arenes and allows corannulene, for example, to perform aromatic substitution reactions as well as addition reactions (well known for the organic chemistry of buckybowls). Yet another oxidation mode could be direct attack of the pentagonal carbon atoms in curved PAH which we have previously explored in fullerenes[37].

\subsection{Interactions between $\mathrm{cPAH}$ and other species}

Permanent dipole-dipole interactions between cPAH and polar molecules could be important for adsorption of species such as water or nitrous oxide onto soot particles. Calculations have found significant interactions between water and corannulene with similar binding strengths to water dimers, $-12.0 \mathrm{~kJ} / \mathrm{mol}$ [38] compared with $-13.2 \mathrm{~kJ} / \mathrm{mol}$ [39] respectively (with water and graphite interaction energies of $-9 \mathrm{~kJ} / \mathrm{mol}$ reported [40]). These interactions could contribute to the uptake of water by soot particles, which has been experimentally observed[41]. The interaction of non-polar molecules with curved PAHs, while not important at soot formation temperatures, could be important for the adsorption of species onto carbon at low temperature and for gas storage applications. Hydrogen absorption onto corannulene is significantly enhanced by dipoleinduced dipole interactions with the binding energy increasing by 59\%[42]. Finally, dispersion interactions of small species, such as gases are enhanced due to the

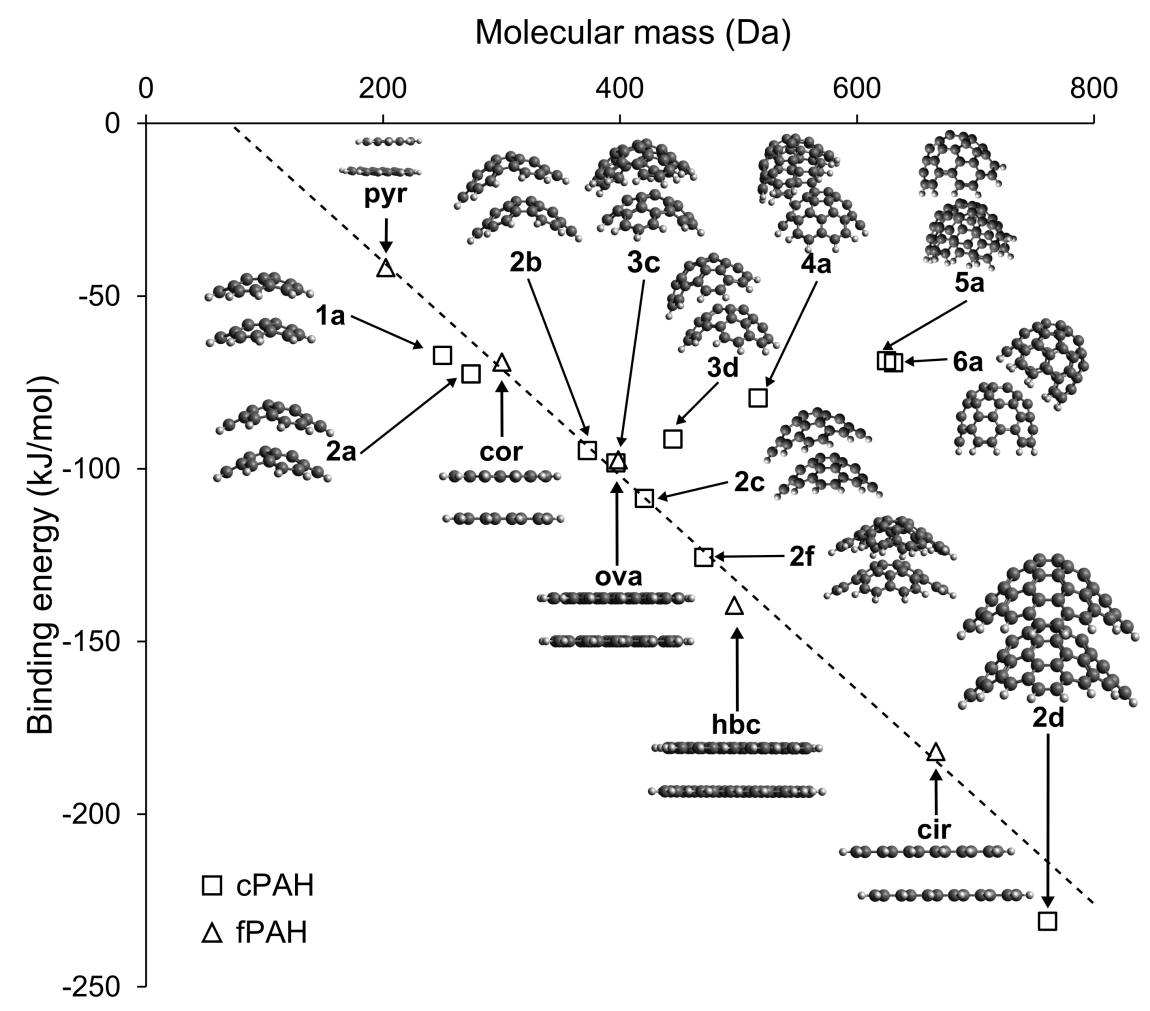

Figure 5: Binding energy of flat and curved PAH molecules as a function of molecular mass. 
decrease in the carbon to adsorbent distance on the concave face of the curved PAH bowl. This has been explored for hydrogen absorption inside nanotubes and nanohorns and could be used to enhance the capacity for gas storage by soot.[42]

The most significant interaction of cPAH with another species will be with charged species. Synthesised cPAHs such as corannulene 1a are known to bind ions strongly (93-289 kJ/mol [43]). Anion binding is enhanced on the concave side by increased interaction with the positive hydrogen atoms and the increased dispersion interactions[44]. Cations can also bind to the concave face by interacting with the small amount of negative charge present (Figure 1), but most significantly they bind via dispersion interactions in this configuration[43]. Binding on the convex face favours binding above the pentagonal ring but can also strongly bond around the rim carbon atoms where charge is concentrated due to the $\mathrm{C}-\mathrm{H}$ bond. Similar binding energies are found between curved and planar PAHs [30, 43]. Curved aromatic molecules differ from planar $\mathrm{PAH}$ in their capacity for ions due to the longer range of the interactions $\left(\propto-1 / r^{2}\right)$. This allows for corannulene to have increased capacity for lithium in battery applications (capacities of 372, and $602 \mathrm{mAh} / \mathrm{g}$ found for graphite and corannulene respectively[45]). Chen and Wang recently considered the stabilisation of clusters of fPAH bound to a chemi-ion. A sandwich complex was found to have significant binding energies - potentially enough for stabilisation at flame temperatures - however, addition of a third fPAH was weakly bound[30]. The binding energy of curved PAH to ions is similar to that of flat PAH[43] but the interaction energy scales as $\propto 1 / r^{2}$, suggesting a longer range to the interactions. Molecular dynamics simulations would be required to further explore this possibility and are beyond the scope of this article. Furthermore, significant work is needed to determine the amount of curved PAH in soot formation to understand the possibility of an ion-induced nucleation mechanism in soot formation.

\section{Conclusion}

The earliest onset of curvature integration in PAH with pentagon integration was found for structures with greater than six rings with at least one pentagonal ring. Acetylene addition was found to increase the curvature to a greater degree than hexagonal rings. Curvature integration is then understood as driven by the $\sigma$-bonding aromatic network while structure planarisation is due to $\pi$-bonding and is only overcome for structures with greater than six rings. The binding energy of curved
PAH dimers was found to be comparable with that of planar PAH molecules due to the contribution from the dipole moment, but steric effects reduced the dispersion interactions leading to structures with greater than three pentagons being significantly lower in binding energy. Finally, the impact of curved PAH on other species and the role of polar aromatics interacting with chemi-ions were considered.

\section{Acknowledgments}

This project is supported by the National Research Foundation (NRF), Prime Minister's Office, Singapore under its Campus for Research Excellence and Technological Enterprise (CREATE) programme.

\section{References}

[1] J. R. McConnell, R. Edwards, G. L. Kok, M. G. Flanner, C. S. Zender, E. S. Saltzman, J. R. Banta, D. R. Pasteris, M. M. Carter, J. D. W. Kahl, 20th-Century Industrial Black Carbon Emissions Altered Arctic Climate Forcing, Science 317 (5843) (2007) 1381-1384. doi:10.1126/science.1144856.

[2] T. C. Bond, S. J. Doherty, D. W. Fahey, P. M. Forster, T. Berntsen, B. J. Deangelo, M. G. Flanner, S. Ghan, B. Kärcher, D. Koch, S. Kinne, Y. Kondo, P. K. Quinn, M. C. Sarofim, M. G. Schultz, M. Schulz, C. Venkataraman, H. Zhang, S. Zhang, N. Bellouin, S. K. Guttikunda, P. K. Hopke, M. Z. Jacobson, J. W. Kaiser, Z. Klimont, U. Lohmann, J. P. Schwarz, D. Shindell, T. Storelvmo, S. G. Warren, C. S. Zender, Bounding the role of black carbon in the climate system: A scientific assessment, Journal of Geophysical Research Atmospheres 118 (11) (2013) 5380-5552. arXiv:arXiv:1011.1669v3, doi:10.1002/jgrd.50171.

[3] P. J. Landrigan, R. Fuller, N. J. R. Acosta, O. Adeyi, R. Arnold, N. N. Basu, A. B. Baldé, R. Bertollini, S. Bose-O'Reilly, J. I. Boufford, P. N. Breysse, T. Chiles, C. Mahidol, A. M. Coll-Seck, M. L. Cropper, J. Fobil, V. Fuster, M. Greenstone, A. Haines, D. Hanrahan, D. Hunter, M. Khare, A. Krupnick, B. Lanphear, B. Lohani, K. Martin, K. V. Mathiasen, M. A. McTeer, C. J. L. Murray, J. D. Ndahimananjara, F. Perera, J. Potočnik, A. S. Preker, J. Ramesh, J. Rockström, C. Salinas, L. D. Samson, K. Sandilya, P. D. Sly, K. R. Smith, A. Steiner, R. B. Stewart, W. A. Suk, O. C. P. van Schayck, G. N. Yadama, K. Yumkella, M. Zhong, The Lancet Commission on pollution and health, The Lancetdoi:10.1016/S0140-6736(17)32345-0.

[4] H. Wang, Formation of nascent soot and other condensed-phase materials in flames, Proceedings of the Combustion Institute 33 (1) (2011) 41 - 67. doi:10.1016/j.proci.2010.09.009.

[5] D. Su, R. Jentoft, J.-O. Müller, D. Rothe, E. Jacob, C. Simpson, Ž. Tomović, K. Müllen, A. Messerer, U. Pöschl, R. Niessner, R. Schlögl, Microstructure and oxidation behaviour of Euro IV diesel engine soot: a comparative study with synthetic model soot substances, Catalysis Today 90 (1-2) (2004) 127-132. doi:10.1016/j.cattod.2004.04.017.

[6] M. L. Botero, D. Chen, S. González-Calera, D. Jefferson, M. Kraft, HRTEM evaluation of soot particles produced by the non-premixed combustion of liquid fuels, Carbon 96 (2016) 459-473. doi:10.1016/j.carbon.2015.09.077. 
[7] E. K. Yapp, C. G. Wells, J. Akroyd, S. Mosbach, R. Xu, M. Kraft, Modelling pah curvature in laminar premixed flames using a detailed population balance model, Combustion and Flame 176 (2017) 172-180.

[8] A. L. Lafleur, J. B. Howard, J. A. Marr, T. Yadav, Proposed fullerene precursor corannulene identified in flames both in the presence and absence of fullerene production, The Journal of Physical Chemistry 97 (51) (1993) 13539-13543. doi:10.1021/j100153a020.

[9] P. Gerhardt, S. Löffler, K. H. Homann, Polyhedral carbon ions in hydrocarbon flames, Chemical Physics Letters 137 (4) (1987) 306-310. doi:10.1016/0009-2614(87)80889-8.

[10] J. W. Martin, R. I. Slavchov, E. K. Y. Yapp, J. Akroyd, S. Mosbach, M. Kraft, The Polarization of Polycyclic Aromatic Hydrocarbons Curved by Pentagon Incorporation: The Role of the Flexoelectric Dipole, The Journal of Physical Chemistry C (2017) acs.jpcc.7b09044doi:10.1021/acs.jpcc.7b09044.

[11] M. L. Botero, E. M. Adkins, S. González-Calera, H. Miller, M. Kraft, PAH structure analysis of soot in a non-premixed flame using high-resolution transmission electron microscopy and optical band gap analysis, Combustion and Flame 164 (2016) 250-258. doi:10.1016/j.combustflame.2015.11.022.

[12] S. L. Shostak, W. L. Ebenstein, J. S. Muenter, The dipole moment of water. I. Dipole moments and hyperfine properties of $\mathrm{H} 2 \mathrm{O}$ and $\mathrm{HDO}$ in the ground and excited vibrational states, The Journal of Chemical Physics 94 (9) (1991) 5875. doi:10.1063/1.460471.

[13] M. J. Frisch, G. W. Trucks, H. B. Schlegel, G. E. Scuseria, M. A. Robb, J. R. Cheeseman, G. Scalmani, V. Barone, G. A. Petersson, H. Nakatsuji, X. Li, M. Caricato, A. Marenich, J. Bloino, B. G. Janesko, R. Gomperts, B. Mennucci, H. P. Hratchian, J. V. Ortiz, A. F. Izmaylov, J. L. Sonnenberg, D. WilliamsYoung, F. Ding, F. Lipparini, F. Egidi, J. Goings, B. Peng, A. Petrone, T. Henderson, D. Ranasinghe, V. G. Zakrzewski, J. Gao, N. Rega, G. Zheng, W. Liang, M. Hada, M. Ehara, K. Toyota, R. Fukuda, J. Hasegawa, M. Ishida, T. Nakajima, Y. Honda, O. Kitao, H. Nakai, T. Vreven, K. Throssell, J. A. Montgomery, J. E. Peralta, F. Ogliaro, M. Bearpark, J. J. Heyd, E. Brothers, K. N. Kudin, V. N. Staroverov, T. Keith, R. Kobayashi, J. Normand, K. Raghavachari, A. Rendell, J. C. Burant, S. S. Iyengar, J. Tomasi, M. Cossi, J. M. Millam, M. Klene, C. Adamo, R. Cammi, J. W. Ochterski, R. L. Martin, K. Morokuma, O. Farkas, J. B. Foresman, D. J. Fox, Gaussian 09, Revision A 02.

[14] M. A. Petrukhina, K. W. Andreini, J. Mack, L. T. Scott, X-ray quality geometries of geodesic polyarenes from theoretical calculations: What levels of theory are reliable?, J. Org. Chem. 70 (14) (2005) 5713-5716. doi:10.1021/jo050233e.

[15] S. Grabowsky, M. Weber, Y. S. Chen, D. Lentz, B. M. Schmidt, M. Hesse, P. Luger, Electron density of corannulene from synchrotron data at $12 \mathrm{~K}$, comparison with fullerenes, Zeitschrift fur Naturforschung - Sect. B J. Chem. Sci. 65 (4) (2010) 452-460. doi:10.1515/znb-2010-0403.

[16] T. S. Totton, A. J. Misquitta, M. Kraft, Assessing the Polycyclic Aromatic Hydrocarbon Anisotropic Potential with Application to the Exfoliation Energy of Graphite, The Journal of Physical Chemistry A 115 (46) (2011) 13684-13693. doi:10.1021/jp208088s.

[17] S. Grimme, Semiempirical GGA-type density functional constructed with a long-range dispersion correction, Journal of Computational Chemistry 27 (15) (2006) 1787-1799. doi:10.1002/jcc.20495.

[18] T. Janowski, P. Pulay, A. A. Sasith Karunarathna, A. Sygula, S. Saebø, Convex-concave stacking of curved conjugated networks: Benchmark calculations on the corannulene dimer, Chemical Physics Letters 512 (4-6) (2011) 155-160. doi:10.1016/j.cplett.2011.07.030.

[19] E. M. Cabaleiro-Lago, B. Fernández, J. Rodríguez-Otero, Dissecting the concave-convex $\pi-\pi$ interaction in corannulene and sumanene dimers: SAPT(DFT) analysis and performance of DFT dispersion-corrected methods, Journal of Computational Chemistry (2017) 1-12doi:10.1002/jcc.25084

[20] C. Pope, J. Marr, J. Howard, Chemistry of fullerenes $\mathrm{C}_{60}$ and $\mathrm{C}_{70}$ formation in flames, The Journal of Physical Chemistry 97 (1993) 11001-11013. doi:10.1021/j100144a018.

[21] R. Whitesides, M. Frenklach, Detailed kinetic Monte Carlo simulations of graphene-edge growth., The journal of physical chemistry. A 114 (2) (2010) 689-703. doi:10.1021/jp906541a.

[22] X. You, R. Whitesides, D. Zubarev, W. A. Lester, M. Frenklach, Bay-capping reactions : Kinetics and influence on grapheneedge growth, Proceedings of the Combustion Institute 33 (1) (2011) 685-692. doi:10.1016/j.proci.2010.05.110.

[23] R. Whitesides, M. Frenklach, Effect of Reaction Kinetics on Graphene-Edge Morphology and Composition, Zeitschrift für Physikalische Chemie 229 (4). doi:10.1515/zpch-2014-0633.

[24] R. Singh, M. Frenklach, A mechanistic study of the influence of graphene curvature on the rate of high-temperature oxidation by molecular oxygen, Carbon 101 (2016) 203-212. doi:10.1016/j.carbon.2016.01.090.

[25] K. H. Homann, Fullerenes and soot formation - New pathways to large particles in flames, Angewandte Chemie, International Edition in English 37 (18) (1998) 2435-2451.

[26] X. Z. Wu, Y. R. Yao, M. M. Chen, H. R. Tian, J. Xiao, Y. Y. Xu, M. S. Lin, L. Abella, C. B. Tian, C.-L. Gao, Q. Zhang, S. Y. Xie, R. B. Huang, L. S. Zheng, Formation of Curvature Subunit of Carbon in Combustion, Journal of the American Chemical Society 138 (30) (2016) 9629-9633. doi:10.1021/jacs.6b04898.

[27] I. Nikiforov, E. Dontsova, R. D. James, T. Dumitric??, Tightbinding theory of graphene bending, Physical Review B - Condensed Matter and Materials Physics 89 (15) (2014) 1-13. doi:10.1103/PhysRevB.89.155437.

[28] J. H. Miller, W. G. Mallard, K. C. Smyth, Intermolecular potential calculations for polycyclic aromatic hydrocarbons, The Journal of Physical Chemistry 88 (21) (1984) 4963-4970. doi: $10.1021 / \mathrm{j} 150665 \mathrm{a} 034$.

[29] T. S. Totton, A. J. Misquitta, M. Kraft, A quantitative study of the clustering of polycyclic aromatic hydrocarbons at high temperatures., Physical chemistry chemical physics 14 (12) (2012) 4081-94. doi:10.1039/c2cp23008a.

[30] D. Chen, H. Wang, Cation- $\pi$ Interactions between Flame Chemi-ions and Aromatic Compounds, Energy \& Fuels 31 (3) (2017) 2345-2352. doi:10.1021/acs.energyfuels.6b02354.

[31] R. Podeszwa, Interactions of graphene sheets deduced from properties of polycyclic aromatic hydrocarbons, The Journal of Chemical Physics 132 (4) (2010) 044704. doi:10.1063/1.3300064.

[32] A. S. Filatov, L. T. Scott, M. A. Petrukhina, $\pi \pi$ Interactions and Solid State Packing Trends of Polycyclic Aromatic Bowls in the Indenocorannulene Family: Predicting Potentially Useful Bulk Properties, Crystal Growth \& Design 10 (10) (2010) 4607-4621. doi:10.1021/cg100898g.

[33] J. S. Chickos, P. Webb, G. Nichols, T. Kiyobayashi, P.-C. Cheng, L. Scott, The enthalpy of vaporization and sublimation of corannulene, coronene, and perylene at $\mathrm{T}=298.15 \mathrm{~K}$, The Journal of Chemical Thermodynamics 34 (8) (2002) 1195-1206. doi:10.1006/jcht.2002.0977.

[34] V. Oja, E. M. Suuberg, Vapor Pressures and Enthalpies of Sublimation of Polycyclic Aromatic Hydrocarbons and Their Derivatives, Journal of Chemical \& Engineering Data 43 (3) (1998) 486-492. doi:10.1021/je9702221. 
[35] P. Grančič, J. W. Martin, D. Chen, S. Mosbach, M. Kraft, Can nascent soot particles burn from the inside?, Carbon 109 (2016) 608-615. doi:10.1016/j.carbon.2016.08.025.

[36] M. a. Dobrowolski, A. Ciesielski, M. K. Cyrański, On the aromatic stabilization of corannulene and coronene, Physical Chemistry Chemical Physics 13 (46) (2011) 20557. doi:10.1039/c1cp21994d.

[37] J. W. Martin, G. J. McIntosh, R. Arul, R. N. Oosterbeek, M. Kraft, T. Söhnel, Giant fullerene formation through thermal treatment of fullerene soot, Carbon 125 (2017) 132-138. doi:10.1016/j.carbon.2017.09.045.

[38] C. Pérez, A. L. Steber, A. M. Rijs, B. Temelso, G. C. Shields, J. C. Lopez, Z. Kisiel, M. Schnell, Corannulene and its complex with water: a tiny cup of water, Phys. Chem. Chem. Phys. 19 (22) (2017) 14214-14223. doi:10.1039/C7CP01506B.

[39] B. E. Rocher-Casterline, L. C. Ch'ng, A. K. Mollner, H. Reisler, Communication: Determination of the bond dissociation energy ( D 0 ) of the water dimer, (H $2 \mathrm{O}) 2$, by velocity map imaging, The Journal of Chemical Physics 134 (21) (2011) 211101. doi:10.1063/1.3598339.

[40] G. R. Jenness, K. D. Jordan, DF-DFT-SAPT Investigation of the Interaction of a Water Molecule to Coronene and Dodecabenzocoronene: Implications for the Water - Graphite Interaction, J. Phys. Chem. C 113 (23) (2009) 10242-10248.

[41] O. B. Popovicheva, N. M. Persiantseva, V. Tishkova, N. K. Shonija, N. A. Zubareva, Quantification of water uptake by soot particles, Environmental Research Letters 3 (2) (2008) 025009. doi:10.1088/1748-9326/3/2/025009.

[42] L. G. Scanlon, P. B. Balbuena, Y. Zhang, G. Sandi, C. K. Back, W. A. Feld, J. Mack, M. A. Rottmayer, J. L. Riepenhoff, Investigation of corannulene for molecular hydrogen storage via computational chemistry and experimentation, Journal of Physical Chemistry B 110 (15) (2006) 7688-7694. doi:10.1021/jp0574403.

[43] R. C. Dunbar, Binding of Transition-Metal Ions to Curved $\pi$ Surfaces: Corannulene and Coronene, The Journal of Physical Chemistry A 106 (42) (2002) 9809-9819. doi:10.1021/jp020313b

[44] H. Chen, W.-Y. Wang, L. Wang, C.-1. Zhu, X.-y. Fang, Y.-q. Qiu, Ion $\pi$ interaction in impacting the nonlinear optical properties of ionbuckybowl complexes, Journal of Molecular Graphics and Modelling 64 (2016) 139-146. doi:10.1016/j.jmgm.2016.01.008.

[45] A. V. Zabula, A. S. Filatov, S. N. Spisak, A. Y. Rogachev, M. A. Petrukhina, A Main Group Metal Sandwich Five Lithium Cations Jammed Between Two Corannulene Tetraanion Decks, Science 333 (6045) (2011) 1008-1011. doi:10.1126/science. 1208686 .

\section{Supplementary Information}

Additional comparison of the B97D functional to B3LYP and to SAPT(DFT) calculations are provided in the Supplementary Information. 